\title{
Two Near-Optimal Layouts for Trusslike Bridge Structures Bearing Uniform Weight between Supports
}

\author{
Jaime Cervera Bravo , Carlos Vázquez Espí , and Mariano Vázquez Espí
}

\begin{abstract}
Although the primary objective on designing a structure is to support the external loads, the achievement of an optimal layout that reduces all costs associated with the structure is an aspect of increasing interest. The problem of finding the optimal layout for bridgelike structures subjected to a uniform load is considered. The problem is formulated following a theory on economy of frame structures, using the stress volume as the objective function and including the selection of appropriate values for statically indeterminate reactions. It is solved in a function space of finite dimension instead of using a general variational approach, obtaining near-optimal solutions. The results obtained with this profitable strategy are very close to the best layouts known to date, with differences of less than $2 \%$ for the stress volume, but with a simpler layout that can be recognized in some real bridges. This strategy could be a guide to preliminary design of bridges subject to a wide class of costs.
\end{abstract}

\section{Introduction}

To bear a uniform weight is one of the fundamental tasks of bridges and other bending structures. When these structures are designed, one of the main aims is to find optimal or at least good structural shapes accounting for all the necessary costs, following the classic rule of thermodynamics to measure efficiency [see, e.g., Clausius (1885)]. This paper will follow the formulation of the design problem stated by Maxwell (1870) and Michell (1904), known as the free loading problem after Cox (1965), or the Maxwell class of problems after Cervera Bravo (1989). Moreover, the general design problem stated by Galilei (1638) to find a structure bearing a given useful load and its own self-weight includes the Maxwell problem as the limiting case in which the structure self-weight is very small and can be neglected [see for details Cervera Bravo and Vázquez Espí (2011) and Antuña and Vázquez Espí (2012)].

The Maxwell approach is clearly different from other problem formulations for the layout optimization, as e.g. the fixed boundary approach (Cox 1965; Hemp 1973) that has received most attention by researchers in the last decades and has had some absolute optima obtained recently (Darwich et al. 2010; Pichugin et al. 2012). In a free loading problem there is no kinematic condition and loads and reactions are known. On the contrary, in a fixed boundary problem there are kinematic conditions and only the loads are known, while the reactions are determined for each feasible solution by standard structural analysis with given constitutive equations for the structural material. Both classes have a common subset of problems, those of the statically determinate kinematic conditions in the latter. And because of this fact, a controversy exists about the meaning of this distinction [e.g., Rozvany (2011) and Vázquez Espí (2013)], which was first noted by Cox (1965, p. 116-117). The fixed boundary formulation has a well-known drawback: "the reactions such as those at fixed supports, are in any case carried by some other bodies acting as structures and the true picture of the economy achieved should include their cost" (Owen 1965, p. 64) because the fixed support cost is different for each solution (Cox 1965). Recently, Rozvany and Sokół (2012) have extended the PragerRozvany theory trying to fill this shortcoming, but it seems that the simple case of a foundation with friction forces is not covered by the new approach (Vázquez Espí 2012). This paper is mainly interested in the free loading formulation because all costs are considered. In a general sense, this approach focuses on the notion of structural design outlined by Cross (1936), which is different from structural analysis.

The plan of exposition is as follows. First, a minimal set of definitions and theorems is introduced for a complete and clear description of the class of design problems with which the paper deals. Second, the class of problems to which the title problem belongs to is described, showing the absolute optimal solutions known up to date for some problems of the class and presenting the near-optimal solutions for the title problem. Third, the process of obtaining the full-plane solution is explained in detail, and then the same method is applied for the half-plane case. In the latter Maxwell problems with given horizontal reactions at the supports are examined, showing how their solutions can be used to select a preliminary bridge layout. Finally the significance of the results is discussed.

\section{Maxwell and Michell Approach to Structural Design}

Definition 1. Maxwell problem: to find a feasible structure for a given set of known, external forces in equilibrium. Each external force must be determined in position, direction and magnitude 
(Maxwell 1870, p. 173, 176-177).The given forces are generally the union of given loads and selected reactions to equilibrate them. The number of reactions can be less than, equal to, or greater than the number of global equilibrium equations (e.g., three in twodimensional space), provided that they are fulfilled by the complete set of given forces. There are no kinematic conditions, i.e., no displacements are imposed (Hemp 1958, p. 2). Neither Maxwell nor Michell used the term reaction, but external force or given force. Maxwell conceived this approach to compare structures that require the same foundation, so its cost can be accounted later because it is constant for all the feasible structures and makes no difference among them. To consider the self-weight always leads to a nonMaxwell problem because the self-weight is not given until a structural solution is considered.

Definition 2. Maxwell structure: Any set of internal forcesdefined by their magnitude $e$ (taking compression as negative) and their two application points, being $\ell$ the distance between them - such that added to the external forces of a structural problem to form a complete set of internal and external forces, this latter satisfies that every subset of all the forces acting at the same point has a null resultant (Maxwell 1870, p. 161). A Maxwell structure can have any number of internal forces for a given problem, i.e., if $\boldsymbol{m}$ is the number of points and $\boldsymbol{n}$ the number of internal forces, there are $2 \boldsymbol{m}$ equilibrium equations in two-dimensional space and $\boldsymbol{n}$ independent variables, and $\boldsymbol{n}$ can be less than, equal to, or greater than $2 m$ provided that the $2 m$ equilibrium equations are fulfilled for the given external forces. To obtain an actual structure from a Maxwell one, additional members or rigid joints can be necessary. Generally, the cost $\mathcal{C}$ of any design is the sum of the cost of foundations $\mathcal{C}_{e}$ - depending on the reactions - and the cost of the structure $\mathcal{C}_{i}$-depending on the internal forces. But for a Maxwell problem with constant reactions, the variation of the cost only depends on the latter, i.e., $\delta \mathcal{C}=\delta \mathcal{C}_{i}$, because $\delta \mathcal{C}_{e}=0$. This is the insight that Maxwell annotated in three short paragraphs of his paper of 1870 and Michell discovered and put in plain mathematics in 1904.

Definition 3. Maxwell number $\mathcal{M}$ of a Maxwell structure: the negative of the virtual work done by the internal forces when the space undergoes a homogeneous deformation that reduces it to a point, $\mathcal{M}=\sum_{i} e_{i} \ell_{i}=\int \boldsymbol{e d} \ell$ (Maxwell 1870, p. 177).

Lemma 4 (Maxwell's Lemma). The Maxwell number of all the structures that solve a given Maxwell problem is constant [Maxwell 1870, p. 177; Michell 1904, Eq. (1); Owen 1965, p. 50]. By the virtual work principle, the internal work of all Maxwell structures equals the work of the given external forces. Hence, the Maxwell number $\mathcal{M}$ is a constant property of each Maxwell problem. This is not the case for fixed boundary problems with statically undetermined kinematic conditions. This lemma is also known as Maxwell's theorem [Hemp 1958, p. 3, Eq. (1)].

Definition 5. Quantity $\mathcal{V}$ or stress volume of a structure: $\mathcal{V}=$ $\sum_{i}\left|\boldsymbol{e}_{i}\right| \ell_{i}=\int|\boldsymbol{e}| \boldsymbol{d} \ell$. Quantity is the name used by Michell [1904, $\mathrm{Eq}$. (3)]. The stress volume denomination arises from the fact that $|\boldsymbol{e}| \ell=|\boldsymbol{\sigma}| \boldsymbol{A} \ell$, with $\boldsymbol{A}$ being the cross-sectional area of a typical truss bar and $\sigma$ its stress, and hence $\mathcal{V}$ can be viewed as the stress volume of the framework. Other denominations have been used: structural work (de Miguel Rodríguez and Aroca 1974), absolute pertinacity (French 1999), and load path (Baker et al. 2013). The stress volume can be separated in the tension $\mathcal{V}^{+}$and compression $\mathcal{V}^{-}$contributions, so that $\mathcal{V}=\mathcal{V}^{+}+\mathcal{V}^{-}$, which will be used in the following.

Corollary 6. The Maxwell number is the difference between stress volumes of tension and compression, $\mathcal{M}=\mathcal{V}^{+}-\mathcal{V}^{-}$ [Maxwell 1870, p. 176; Michell 1904, Eq. (1)].
Corollary 7. In any Maxwell problem, any feasible variation of a feasible solution is such that the variation of tension volume equals the compression one: $\delta \mathcal{V}^{+}=\delta \mathcal{V}^{-}$(Parkes 1965, p. 163).

Lemma 8 (Michell's Lemma). For any $\operatorname{cost} \mathcal{C}_{i}$ defined as $\mathcal{C}_{i}=$ $\boldsymbol{k}_{+} \mathcal{V}^{+}+\boldsymbol{k}_{-} \mathcal{V}^{-}$with $\boldsymbol{k}_{+} \geq 0, \boldsymbol{k}_{-} \geq 0$ and $\boldsymbol{k}_{+}+\boldsymbol{k}_{-}>0, \delta \boldsymbol{C}_{\boldsymbol{i}}=0 \Leftrightarrow$ $\delta \mathcal{V}=0$ holds for a Maxwell problem, i.e., the solution of minimal $\mathcal{V}$ will be of minimal $\mathcal{C}_{i}$ [Michell 1904, Eq. (3); Hemp 1958, p. 4, Eq. (7)]. The geometrical volume of a fully-stressed truss is $\boldsymbol{V}=\mathcal{V}^{+} / \boldsymbol{f}_{+}+\mathcal{V}^{-} / f_{-}$, where $\boldsymbol{f}_{+}$and $\boldsymbol{f}_{-}$are the allowable stress of tension and compression, respectively. In fact, $1 / f$ is the geometrical volume per unit of stress volume, i.e., $1 / f_{+}, 1 / f_{-}$are unitary costs. A similar definition can be given for structural weight (being the unitary costs $\gamma_{+} / f_{+}, \gamma_{-} / f_{-}$and denoting $\gamma$ the specific weight of used materials) and embodied energy of materials. A key difference between free loading and fixed boundary problems arises from this lemma. In the former, an optimal Maxwell structure has the same shape for any $\mathcal{C}_{i}(\operatorname{Cox} 1965, \mathrm{p} .116)$. In the latter, the shape of the optimum solution depends on the proportion between unitary costs of tension and compression (Srithongchai and Dewhurst 2003; Pichugin et al. 2012). Although Michell (and many other authors later) developed the layout theory considering the geometric volume as the functional to be minimized, in the sequel the stress volume $\mathcal{V}$ will be used. Any other cost $\mathcal{C}_{i}$ will be computed as

$$
\begin{aligned}
\mathcal{C}_{\boldsymbol{i}} & =\frac{1}{2}\left[\left(\boldsymbol{k}_{+}+\boldsymbol{k}_{-}\right) \cdot \mathcal{V}+\left(\boldsymbol{k}_{+}-\boldsymbol{k}_{-}\right) \cdot \mathcal{M}\right] \\
& =\frac{\boldsymbol{k}_{+}+\boldsymbol{k}_{-}}{2} \cdot \mathcal{V} \cdot\left(1+\frac{\boldsymbol{k}_{+}-\boldsymbol{k}_{-}}{\boldsymbol{k}_{+}+\boldsymbol{k}_{-}} \cdot \frac{\mathcal{M}}{\mathcal{V}}\right)
\end{aligned}
$$

\section{Michell's Theorems}

Let $\Delta$ be a finite, strictly positive strain. Let $\mathcal{D}$ be the set of bounded, continuous displacement fields $d$ such that the strain $\varepsilon^{d}$ of the field $d$ at all points and directions of the considered domain, $\Omega$, fulfills $\left|\varepsilon^{d}\right| \leq \Delta_{d}$. Let $\mathcal{S}$ be the set of all Maxwell structures for a Maxwell problem enclosed into $\Omega$.

Theorem 9. (Michell's first theorem)

$$
\forall(\boldsymbol{d}, \boldsymbol{A}) \in \mathcal{D} \times \mathcal{S}: \frac{W^{d}}{\Delta_{d}} \leq \mathcal{V}(\boldsymbol{A})
$$

where $\boldsymbol{W}^{\boldsymbol{d}}$ is the virtual work of the given external forces of the Maxwell problem when the domain $\mathbf{\Omega}$ undergoes the displacement field $\boldsymbol{d}$ (Michell 1904, p. 590). Hence, if $\mathcal{L}$ is defined as

$$
\mathcal{L}=\sup _{d}\left\{\frac{W^{d}}{\Delta_{d}}: d \in \mathcal{D}\right\} \leq \inf _{A}\{\mathcal{V}(A): A \in \mathcal{S}\}
$$

$\mathcal{L}$ is a lower bound of $\mathcal{V}$, named "the limit of economy of material" by Michell (1904, p. 591).

Theorem 10. (Michell's second theorem): If a pair $(\boldsymbol{T}, \boldsymbol{M}) \in$ $\mathcal{D} \times \mathcal{S}$ exists for $\boldsymbol{\Omega}$, such that $\boldsymbol{\varepsilon}_{i}^{T} \boldsymbol{e}_{i}^{\boldsymbol{M}}=\boldsymbol{\Delta}_{\boldsymbol{T}}\left|\boldsymbol{e}_{i}^{\boldsymbol{M}}\right|$ for every member $\boldsymbol{i}$, then "the truss $\boldsymbol{M}$ attains the limit of economy of material" in $\boldsymbol{\Omega}, \mathcal{V}(\boldsymbol{M})$ "is a minimum, and consequently from [Michell's lemma] the volume of material in the frame $\boldsymbol{M}$ is also a minimum." (Michell 1904 , p. 591). Hence, Michell's optimality criterion in $\boldsymbol{\Omega}$ requires: - a finite bound strictly positive $\Delta_{T}$ for the field $T$, and

- $\varepsilon_{i}^{T} e_{i}^{M}=\Delta_{T}\left|e_{i}^{M}\right|$ holds on all members of the structure $M$. Because Michell does not show any proof of the existence of a pair $(T, M)$ for every set of given external forces in equilibrium, he only considers his criterion as a sufficient condition (Michell 1904, p. 589). In spite of a sustained research effort on this subject, it has not been proven that Michell's criterion is also a necessary 
condition. The best result known to date is that although a maximizer and minimizer pair for Eq. (3) always exists [Bouchitté et al. 2008, Eqs. (2.22) and (2.24) and Proposition 2.1], the minimizer of the right-hand side of Eq. (3) "may not be a Michell truss" (W. Gangbo, personal communication, 2012). Moreover, Bouchitté et al. (2008, $\$ 3.2$ and Theorem 3.1) have reformulated the original problem so that only Michell trusses belong to the counterpart of $\mathcal{S}$ in Eq. (3), redefining the counterpart of $\mathcal{D}$ accordingly. Next, they have proved that the infima of both formulations are equal. Moreover, "if we could prove existence of a minimizer in (3.6) [ibidem], we will use the optimum [Radon measure] $\gamma$ to construct a minimizer $\sigma^{\circ}$ which we know will be a Michell truss" (W. Gangbo, personal communication, 2012). Bouchitté et al. (2008, p. 1601-1602, Problem $5.1)$ conclude saying, "we strongly believe that our approach could be a useful tool to investigate the properties of optimal structures. However, it is still necessary to prove the existence of a minimizing measure [for the new formulation]." This argument refers only to Michell's original criterion for Maxwell problems. The frequently and unfortunately equal named criteria for fixed boundary problems are different for each cost, e.g., Hemp's criterion for minimum volume [1973, Eq. (4.3)] or Prager's criterion for minimum weight (Srithongchai and Dewhurst 2003). As the adjective Michell is nowadays overloaded, remember that this paper uses Michell solution to refer to a Maxwell structure that fulfills Michell's criterion (Theorem 10), i.e., an absolute optimum solution for a given Maxwell problem. However, Michell net is used in its usual sense, i.e., to refer to layouts, frames, or Maxwell structures that follow, fully or partially, the geometrical conditions derived by Michell (1904, p. 591-594) from his second theorem, even if there is no proof that they fulfill Michell's criterion.

Definition 11. Michell number $v$ of a structure: The dimensionless ratio between the stress volume and the product of the useful load and the size of the problem (Cervera Bravo and Vázquez Espí 2011; McConnel 1974, quantity $k$ ).

\section{Bridge Class of Design Problems}

The bridge problem is the Maxwell problem of equilibrating a uniform weight $w$ over a horizontal length $L$ with vertical forces (reactions) in the load line. Depending on the number $S$ of suitable reactions and their relative distances, there are different problems, so it is preferable to refer to the bridge class of problems (Fig. 1). All have the same size $L$ and useful load $w L$, so the Michell number of every solution is computed as $v=\mathcal{V} /\left(w L^{2}\right)$.

For $S=1$, the vertical reaction is equal to $w L$ acting at midspan, so there is a unique problem [Fig. 1(a)]. The Michell solutions for

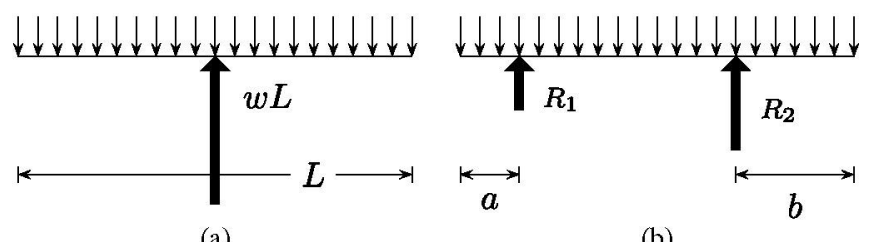

(a)

(b)
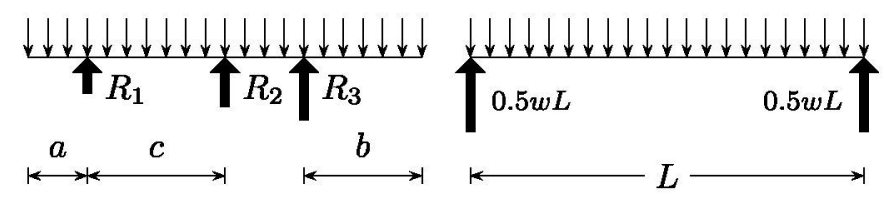

(c)

(d)

Fig. 1. Maxwell problems in the bridge class: (a) $S=1$; (b) $S=2$; (c) $S=3$; (d) $S=2, a=b=0$ the full plane or the half plane are known after the Michell solution for three parallel forces (Fig. 2) [Parkes 1965, p. 177, Fig. 102(d)].

For $S=2$, the sum of the two vertical reactions must be equal to $w L$, but their magnitudes depend on their relative position [Fig. 1(b)]. Each pair of values $a, b$ with $L-a-b>0$ and $a \leq$ $b$ defines a different Maxwell problem. This case covers a fairly large subset of real bridges. No Michell solutions are known to date.

For $S=3$, there are four degrees of freedom to define a Maxwell problem: the positions of supports, $a, b, c$, and the magnitude of one of the reactions, [Fig. 1(c)]. The number of degrees of freedom to define a Maxwell problem will increase with $S$. Thereafter, each value of $S$ represents a new set of Maxwell problems.

\section{Solutions for the $S=2, a=b=0$ Problem}

The results for the case $S=2$, with $a=b=0$, i.e., with the reactions at the edges of $L$, are presented [Fig. 1(d)]. The traditional solutions follow from parabolic curves and vertical hangers (label P), with a horizontal tie in the half-plane case (Table 1). On the contrary, the investigation is around a basic layout that consists of a nonparabolic arch with oblique hangers $(\mathrm{AOH})$, with two versions for both full and half plane [Table 1 and Figs. 3(c and f)]. They follow from a layout family whose first solution was obtained by W. J. Supple and published by Hemp (1973, p. 21) and later proposed by McConnel (1974, Figs. 5 and 6, p. 889) as simpler approximations of his own proposed Michell nets (p. 897). Although McConnel only worked with trusses of finite numbers of nodes-with linear programming (LP) and non-LP algorithms-he predicted with an extrapolation method the value 0.75779 for $v=\mathcal{V} / w L^{2}$ in the fullplane case, which agrees with the result in this paper, 0.75800 , from numerical variational calculus. McConnel predicts slightly better values for his proposed Michell nets (label M), 0.75490 in the full-plane case [Table 1 and Figs. 3( $a$ and d)].

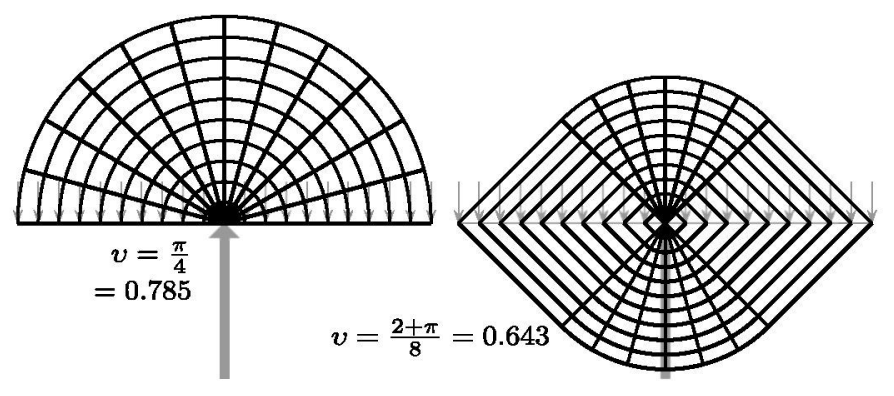

(a)

(b)

Fig. 2. Michell solutions for one support: (a) half-plane solution; (b) full-plane solution

Table 1. Solutions for $S=2$ and $a=b=0$

\begin{tabular}{lccc}
\hline$\Omega$ & Label & $v=\mathcal{V} /\left(w L^{2}\right)$ & $H / L$ \\
\hline Half plane & P,h & 1.155 & 0.433 \\
& AOH, h & 0.98468 & 0.44191 \\
& SA,h & 0.97431 & 0.45535 \\
Full plane & M,h & $\mathbf{0 . 9 7 7 7 6}$ & - \\
& P,f & 0.817 & 0.612 \\
& AOH, f & 0.75800 & 0.63345 \\
& SA,f & 0.75714 & 0.63433 \\
& M,f & $\mathbf{0 . 7 5 4 9 0}$ & - \\
\hline
\end{tabular}

Note: $\mathrm{AOH}=$ arch and oblique hangers; $f=$ full-plane case; $h=$ half-plane case; $\mathrm{M}=\mathrm{McC}$ Connel estimate (no actual layout here); $\mathrm{P}=$ parabolic arch and vertical hangers; $\mathrm{SA}=$ layout from simulated annealing search. 
Hemp (1974) presents a Michell net for the fixed boundary version of the upper half plane problem (with two fixed supports), corresponding to $v=0.78887$, while McConnel predicts 0.78787 , and recently Pichugin et al. (2012) predicted 0.78791, so the predicted values by McConnel can be accepted as sound estimates for stress volume optima in all cases and are thus bolded in Table 1 (label M).

There is also a search with the simulated annealing (SA) algorithm [e.g., Vázquez Espí and Vázquez Espí (1997)] [Table 1 and Figs. 3(b and e)] (SA trusses data-node coordinates and bar nodes-available at http://habitat.aq.upm.es/gi/mve/dt/). As in the algorithms used by McConnel, the SA solutions are of discrete nature. The original uniform weight $w$ is represented by $M-1$ inner point loads of magnitude $w L / M$ uniformly distributed along the span, with $M \in[15,40]$. For each considered $\Omega$, there is a search for symmetrical and nonsymmetrical solutions with a number of nodes $N$ in the interval $[M+2, p \cdot M]$ where $p \in\{3,4,5,6,8\}$. Although the upper bound for $N$ could seem small (e.g., 320), as no ground structure is used so the positions of this small, variable number of nodes and their connectivity change during the search to explore the whole space of feasible solutions without any other restriction.

In both domains, the SA solutions slightly improve the stress volume of the $\mathrm{AOH}$ layouts. The best $\mathrm{SA}, \mathrm{h}$ solution is similar to the Michell net proposed by McConnel and its stress volume is slightly smaller than McConnel's optimum estimate (Table 1). The main difference between $\mathrm{AOH}$ and the better SA layouts occurs near the supports, where the SA solutions show Michell nets, strongly suggesting that Michell solutions could exist in both cases, although only in the half-plane case can improvement of the stress volume of the $\mathrm{AOH}$ layout up to approximately $1 \%$ be ensured. The solutions obtained by J. Hernando García (personal communication, 2011) using LP techniques have been at the authors' disposal, and his best results confirm McConnel's estimates, the presence of oblique hangers and Michell nets near supports, and the probable existence of Michell solutions.

\section{AOH Full-Plane Layout}

Consider a quarter of the layout [Figs. 3(f) and 4]. At the horizontal position $\chi L$, with $\chi \in[0,1 / 2]$, the direction and length of the hanger are, respectively, $\alpha(\chi)$ and $c(\chi)$. Its upper extreme defines a point of the arch of coordinates

$$
\boldsymbol{X}=L(x, y)^{T}=[L \chi+c \sin (\alpha), c \cos (\alpha)]^{T}=L(\chi+y \tan \alpha, y)^{T}
$$

With the notation $t(\chi)=\tan \alpha(\chi),(\cdot)^{\prime}=\mathbf{d}(\cdot) / \mathrm{d} \chi$, the geometric conditions are

$\boldsymbol{X}=L(x, y)^{T}=L(\chi+y t, y)^{T}, \quad \boldsymbol{X}^{\prime}=L\left(x^{\prime}, y^{\prime}\right)^{T}=L\left[1+(y t)^{\prime}, y^{\prime}\right]^{T}$

The equilibrium condition at the bottom of the hanger defines its internal force, $\boldsymbol{P}=\left(P_{h}, P_{v}\right)^{T}$ with $P_{h}=w t / 2$, and $P_{v}=w / 2$. The shape of the arch, $X(\chi)$, is determined by its internal force, $\left(N_{h}, N_{v}\right)^{T}$

$$
\frac{N_{h}}{N_{v}}=-\frac{d x}{d y}=-\frac{x^{\prime}}{y^{\prime}}
$$

The equilibrium of the joint of the hanger and the arch determines the variation of the internal force of the latter

$$
d N_{h}=-P_{h} L d \chi, d N_{v}=P_{v} L d \chi
$$

Now, using the rotational equilibrium condition of one-half of the structure at the vertical axis of symmetry

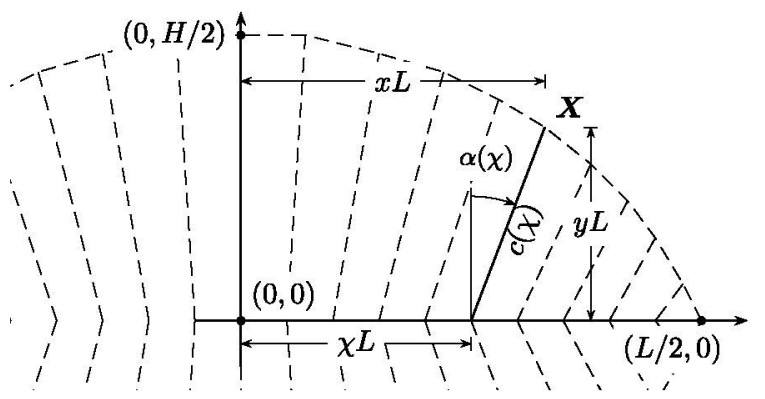

Fig. 4. Quarter of the AOH layout

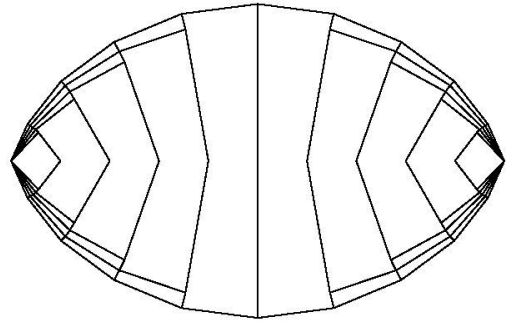

(a)

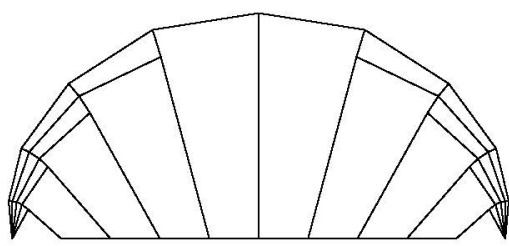

(d)

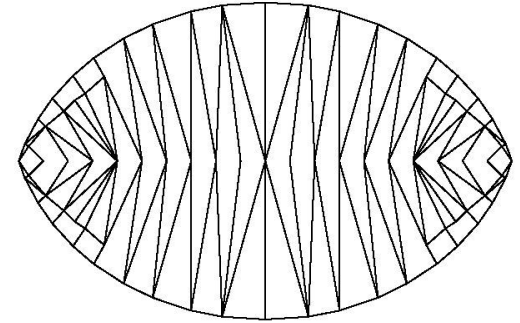

(b)

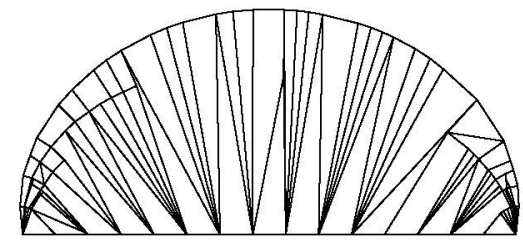

(e)

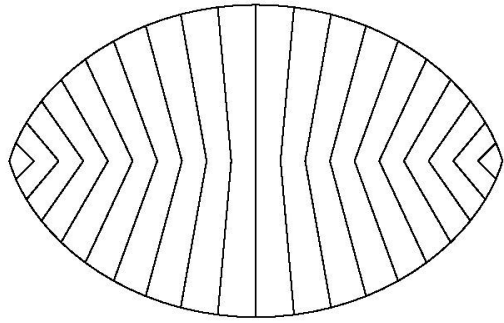

(c)

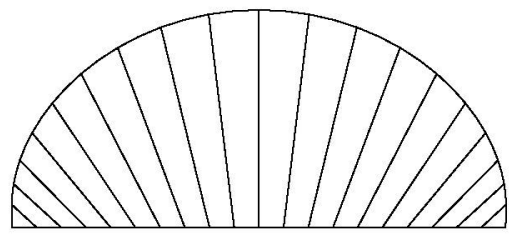

(f)

Fig. 3. Proposed Michell nets by McConnel, best SA trusses and optimal AOH layouts: (a) full-plane Michell net; (b) best SA,f truss, $M=20$, $N=76$; (c) AOH,f layout; (d) half-plane Michell net; (e) best SA,h truss, $M=15, N=96$; (f) AOH,h layout 


$$
N_{h}(0)=\frac{w L^{2} / 8}{2 y(0) L}=\frac{w L}{2} \frac{1}{4 h}=\frac{w L}{2} \frac{\lambda}{4}
$$

where $h=2 y(0)$ is the inverse of the global slenderness $\lambda=L / H$. By integrating Eq. (7)

$$
\begin{aligned}
& N_{h}(\chi)=N_{h}(0)-\int_{0}^{\chi} P_{h} L d u=\frac{w L}{2}\left[\frac{\lambda}{4}-\int_{0}^{\chi} t(u) d u\right] \\
& N_{v}(\chi)=\int_{0}^{\chi} P_{v} L d u=\frac{w L}{2} \chi
\end{aligned}
$$

Now compute the stress volume of one quadrant, $\mathcal{V}_{c}$. Because $\mathcal{V}=\int|e| d s$ and $|e|$ and $d s$ are the modulus of parallel vectors, its product can be computed in terms of their horizontal and vertical components, hence $\mathcal{V}=\int\left|e_{x}\right|\left|x^{\prime}\right| d \chi+\int\left|e_{y}\right|\left|y^{\prime}\right| d \chi=\mathcal{V}^{=}+\mathcal{V}^{\|}$. Furthermore, each quantity can be decomposed in hangers $\left(\mathcal{V}_{c}^{+=}, \mathcal{V}_{c}^{+\mid}\right)$and arch contributions $\left(\mathcal{V}_{c}^{-=}, \mathcal{V}_{c}^{-\|}\right)$according to

$$
\begin{aligned}
\mathcal{V}_{c} & =\mathcal{V}_{c}^{+=}+\mathcal{V}_{c}^{+\|}+\mathcal{V}_{c}^{-=}+\mathcal{V}_{c}^{-\|} \\
\mathcal{V}_{c}^{+=} & =\frac{w L^{2}}{2} \int_{0}^{1 / 2} t^{2} y d \chi \\
\mathcal{V}_{c}^{+\|} & =\frac{w L^{2}}{2} \int_{0}^{1 / 2} y d \chi \\
\mathcal{V}_{c}^{-}= & =\frac{w L^{2}}{2} \int_{0}^{1 / 2}\left[\frac{\lambda}{4}-\int_{0}^{\chi} t(u) d u\right]\left(1+t y^{\prime}+t^{\prime} y\right) d \chi \\
\mathcal{V}_{c}^{-\|} & =\frac{w L^{2}}{2} \int_{0}^{1 / 2} \chi\left(-y^{\prime}\right) d \chi
\end{aligned}
$$

The absolute value operator is not needed because all integrands are positive for $\chi \in[0,1 / 2]$. For example, if the thrust component $N_{h}$ changes sign, because $y^{\prime}<0$ it follows from Eq. (6) that $x^{\prime}$ does as well, so that the product $N_{h} x^{\prime}$ will be positive everywhere. The arch terms can be integrated by parts, taking into account that $t(0)=0$ and $y(1 / 2)=0$, to obtain

$$
\begin{aligned}
& \mathcal{V}_{c}^{-}=\frac{w L^{2}}{2} \int_{0}^{1 / 2}\left[\frac{\lambda}{4}-\int_{0}^{\chi} t(u) d u+t^{2} y\right] d \chi \\
& \mathcal{V}_{c}^{-\|}=\frac{w L^{2}}{2} \int_{0}^{1 / 2} y d \chi
\end{aligned}
$$

Finally, the minimum stress volume is given by

$$
\min _{y, t, \lambda} \mathcal{V}=2 w L^{2} \min _{y, t, \lambda} \int_{0}^{1 / 2}\left[2 y\left(t^{2}+1\right)+\frac{\lambda}{4}-\int_{0}^{\chi} t(u) \mathrm{d} u\right] \mathrm{d} \chi
$$

Hence the functions $y(\chi), t(\chi)$ and the slenderness $\lambda$ that solve the last equation fulfilling the condition of Eq. (6) determine the optimal geometry of the $\mathrm{AOH}$ layout. This problem can be simplified because it is possible to obtain a closed form for $y(\chi)$ as follows. Eqs. (5) and (6) can be written as $y^{\prime} N_{h}+\left[1+(y t)^{\prime}\right] N_{v}=0$, which can be written taking into account Eq. (9) in the form

$$
\{\chi\}+\left\{\chi y t^{\prime}+y^{\prime}\left[\frac{\lambda}{4}-\int_{0}^{\chi} t(u) \mathrm{d} u+\chi t\right]\right\}=0
$$

Because this expression is the sum of two exact differentials, it can be integrated explicitly to get

$$
\frac{1}{2} \chi^{2}+y\left[\frac{\lambda}{4}-\int_{0}^{\chi} t(u) d u+\chi t\right]=\frac{1}{8}
$$

where the initial condition $y(0)=1 / 2 \lambda$ has been used. From Eq. (14), a closed form for $y(\chi)$ can be obtained. Finally, there is the following change of notation: $\tau=\int_{0}^{\chi} t(u) \mathrm{d} u-\lambda / 4$, $\tau^{\prime}=t=\tan \alpha$, with $\tau(0)=-\lambda / 4, \tau^{\prime}(0)=0$. The closed form of $y(\chi)$ can be written as

$$
y(\chi)=\frac{1-4 \chi^{2}}{8\left(\chi \tau^{\prime}-\tau\right)}
$$

Therefore the problem Eq. (12) subject to Eq. (6) can be written as

$$
\mathcal{V}_{\mathrm{opt}}^{\mathrm{AOH}, f}=2 w L^{2} \min _{\tau} \int_{0}^{1 / 2}\left[\frac{\left(1-4 \chi^{2}\right)\left(\tau^{2}+1\right)}{4\left(\chi \tau^{\prime}-\tau\right)}-\tau\right] d \chi
$$

For the sake of brevity, the solution of Eq. (16) will be shown in a reduced function space because a general, variational approach leads to a very similar solution. Specifically, $\tau(\chi)$ will be obtained in the space of polynomial functions of even powers of $\chi$. Let $\tau_{n}(\chi)=\sum_{i=0}^{n} z_{i} \chi^{2 i}$, where $z_{i}(i=0, \ldots, n)$ are real coefficients. Introducing $\tau_{n}(\chi)$ in Eq. (16) creates an expression for $\mathcal{V}$ depending on the coefficients $z_{i}$. The equations $\partial \mathcal{V} / \partial z_{i}=0$ determine the values of $\left(z_{0}, \ldots\right)$ that minimize $\mathcal{V}$ for each selected $n$. Some solutions are shown in Table 2.

\section{AOH Half-Plane Layout}

When the domain $\Omega$ is the upper half plane, a tie between supports is required to equilibrate the horizontal component of hangers and arch across the load line. The internal force in the hanger is $P_{h}=w t, P_{v}=w$, and the internal force in the arch is

$$
N_{h}(\chi)=w L\left[\frac{\lambda}{8}-\int_{0}^{\chi} t(u) d u\right], \quad N_{v}(\chi)=w L \chi
$$

Now $y(0)=h=1 / \lambda$. The internal force in the tie is $T(\chi)=N_{h}(\chi)$. From previous results, it was apparent that both $T$ and $N_{h}$ could change the sign near the supports, and then the layout width would be greater than the span $L$. In the sequel $\chi_{\mathrm{c} r}$ denote the value of $\chi$ for which $T(\chi)=0$.

As previously, compute the stress volume of one half, $\mathcal{V}_{h}$. For the tie

$$
\mathcal{V}_{h}^{\mathrm{tie}}=\int_{0}^{1 / 2}|T(\chi)| L d \chi=\int_{0}^{\chi_{c r}} N_{h} L d \chi-\int_{\chi_{c r}}^{1 / 2} N_{h} L d \chi
$$

The rest of the terms can be obtained from the corresponding terms of $\mathcal{V}_{c}$ in Eqs. (10) or (11), but multiplying by two and substituting $\lambda / 4$ by $\lambda / 8$. Therefore, with $\tau=\int_{0}^{\chi} t(u) d u-\lambda / 8$, the half-plane geometry is the solution of

$$
\mathcal{V}_{\mathrm{opt}}^{\mathrm{AOH}, h}=4 w L^{2} \min _{\tau, \chi_{c r}}\left[\int_{0}^{1 / 2} \frac{\left(1-4 \chi^{2}\right)\left(\tau^{2}+1\right)}{8\left(\chi \tau^{\prime}-\tau\right)} d \chi-\int_{0}^{\chi_{c r}} \tau d \chi\right]
$$

Some polynomial solutions $\tau_{n}(\chi)$ are shown in Table 3 . The coefficients $z_{i}$ are determined as previously, but now a new equation must be introduced for $\chi_{\mathrm{c} r}, \partial \mathcal{V} / \partial \chi_{\mathrm{cr}}=0$. However, note

Table 2. Polynomial Solutions $\tau_{n}$ for the Full-Plane Domain

\begin{tabular}{cccccccr}
\hline & & & & & $\alpha(0.5)$ \\
$n$ & $v$ & $h=1 / \lambda$ & $z_{0}$ & $z_{1}$ & $z_{2}$ & $z_{3}$ & $(\mathrm{deg})$ \\
\hline 0 & 0.81650 & 0.61237 & $-1 / \sqrt{6}$ & - & - & - & 0.00 \\
2 & 0.75800 & 0.63364 & -0.39455 & 0.86111 & 0.57924 & - & 49.01 \\
3 & 0.75800 & 0.63345 & -0.39466 & 0.87357 & 0.40757 & 0.55372 & 49.75 \\
\multicolumn{7}{l}{ Variational, numerical solution: $v=0.75800$ and $h=0.63339$} \\
\hline
\end{tabular}


that in the case of a constant function, $\tau_{0}, \chi_{\mathrm{cr}}=1 / 2$ because $\tau_{0}^{\prime}(\chi)=0$, that is, the hangers are vertical and $T(\chi)$ is constant.

\section{Extension to Cases with Horizontal Reactions}

New Maxwell problems can be obtained from the latter simply by adding a pair of opposite horizontal reactions in the edges. If these reactions were free of cost, it is clear that they could reduce the stress volume of the tie and hence $\mathcal{V}$. This is precisely the case of friction forces between foundations and ground (Bow 1873, p. 69-71). Let the horizontal reaction magnitude be given as a fraction $\phi$ of the vertical one, $R=\phi w L / 2$, hence $\mathcal{M}=-\phi w L^{2} / 2$ from Definition 3. Now the internal force in the tie is $T(\chi)=N_{h}(\chi)-R$ and its stress volume is given by

$$
\begin{aligned}
\mathcal{V}_{h}^{\text {tie }}= & \int_{0}^{1 / 2}|T(\chi)| L d \chi=\int_{0}^{\chi_{c r}} N_{h} L \mathrm{~d} \chi-\int_{\chi_{c r}}^{1 / 2} N_{h} L \mathbf{d} \chi \\
& +\left(\frac{1}{4}-\chi_{c r}\right) \phi w L^{2}
\end{aligned}
$$

where $N_{h}$ is given by Eq. (17). Therefore, with $\tau=$ $\int_{0}^{\chi} t(u) \mathrm{d} u-\lambda / 8$, the $\mathrm{AOH}$ half-plane geometry with horizontal reactions is the solution of

$$
\begin{aligned}
\mathcal{V}_{\mathrm{opt}}^{\mathrm{AOH}, h}(\phi)= & 4 w L^{2} \min _{\tau, \chi_{c r}}\left[\int_{0}^{1 / 2} \frac{\left(1-4 \chi^{2}\right)\left(\tau^{\prime 2}+1\right)}{8\left(\chi^{\prime}-\tau\right)} d \chi-\int_{0}^{\chi_{c r}} \tau d \chi\right. \\
& \left.+\frac{1}{2} \phi\left(\frac{1}{4}-\chi_{c r}\right)\right]
\end{aligned}
$$

A first inquiry is about the optimal $\phi$, i.e., to solve the problem

$$
\min _{\phi} \mathcal{V}_{\mathrm{opt}}^{\mathrm{AOH}, h}(\phi)
$$

The optimal $\phi$ is found to be 0.738 with $v_{\text {opt }}=0.801$. These values can be compared with those of the solution of
Hemp (1974) for the fixed boundary version of the problem because in both cases the optimal horizontal reaction is searched. This Michell net corresponds to $\phi=0.739$ and $v_{\text {opt }}=0.789$, i.e., the solution of this paper will have $1.5 \%$ more geometrical volume for equal tension and compression allowable stresses according to Eq. (1).

The relationship between $\phi$ and the friction coefficient $\mu$ depends on the weight of the foundation $P$. From the Coulomb theory, $R=\mu(P+w L / 2)$ and

$$
\phi(P, \mu)=\frac{R}{\frac{1}{2} w L}=\mu\left(1+\frac{P}{\frac{1}{2} w L}\right)
$$

Only with a soil with more strength than the structural material can $P \approx 0$ be expected. Hence, it will generally be $\phi>\mu$. The soil properties and the foundation shape will require a minimum foundation weight $P_{\min }$ to bear the vertical reaction, $w L / 2$, so it can count with a free-of-cost fraction $\phi\left(P_{\min }, \mu\right)$. For the cases in which this value is smaller than 0.738 , the solution can be obtained from Eq. (21) (Table 4 and Fig. 5).

Furthermore, depending on the costs of foundations and structure materials, it could be convenient to invest in $P$ to increase $\phi$. Let $c_{e}, c_{+}, c_{-}$be the weight-specific costs of foundation, tension, and compression materials, respectively. The unitary costs of the stress volume are $k_{+}=c_{+} \gamma_{+} / f_{+}=c_{+} / \mathcal{S}_{+}$and $k_{-}=$ $c_{-} \gamma_{-} / f_{-}=c_{-} / \mathcal{S}_{-}$, where $\mathcal{S}=f / \gamma$ is the structural scope, a characteristic length of the structural material. Then the total cost for

\begin{tabular}{|c|c|c|c|c|c|c|c|c|c|c|}
\hline$n$ & $v$ & $h$ & $z_{0}$ & $z_{1}$ & $z_{2}$ & $z_{3}$ & $z_{4}$ & $\chi_{\mathrm{cr} r}$ & $\alpha\left(\chi_{\mathrm{cr} r}\right)$ & $\alpha(0.5)$ \\
\hline 0 & 1.15470 & 0.43301 & $-1 / 2 \sqrt{3}$ & - & - & - & - & - & - & - \\
\hline 1 & 0.98515 & 0.44012 & -0.28401 & 1.32299 & - & - & - & 0.46333 & 50.80 & 52.92 \\
\hline 2 & 0.98514 & 0.44031 & -0.28389 & 1.31111 & 0.06018 & - & - & 0.46305 & 51.04 & 53.29 \\
\hline 4 & 0.98468 & 0.44191 & -0.28286 & 1.19293 & 1.08521 & 2.41839 & -21.78533 & 0.46247 & 46.43 & 39.60 \\
\hline
\end{tabular}
optimal $\mathrm{AOH}$ geometries with given $P$ is

$$
\mathcal{C}(P)=2 c_{e} P+\frac{1}{2}\left[\left(k_{+}+k_{-}\right) \cdot \mathcal{V}_{\mathrm{opt}}^{\mathrm{AOH}, h}(\phi)+\left(k_{+}-k_{-}\right) \cdot \mathcal{M}(\phi)\right]
$$

\begin{tabular}{|c|c|c|c|c|c|c|c|c|c|c|}
\hline$\phi$ & $v$ & $h$ & $z_{0}$ & $z_{1}$ & $z_{2}$ & $z_{3}$ & $z_{4}$ & $\chi_{\mathrm{c} r}$ & $\alpha\left(\chi_{\mathrm{cr} r}\right)$ & $\alpha(0.5)$ \\
\hline 0.25 & 0.88905 & 0.415 & -0.30099 & 1.00741 & 1.94770 & -16.44114 & 31.88772 & 0.41590 & 35.75 & 41.72 \\
\hline 0.50 & 0.82341 & 0.373 & -0.33540 & 0.86110 & -1.31500 & 0.73013 & 7.93534 & 0.34203 & 23.45 & 39.91 \\
\hline 0.738 & 0.80096 & 0.317 & -0.39432 & 0.49063 & -2.33128 & 17.57025 & -34.15451 & 0.25000 & 10.53 & 25.86 \\
\hline
\end{tabular}

and the minimum condition is

Table 3. Polynomial Solutions $\tau_{n}$ for the Half-Plane Domain

Table 4. Polynomial Solutions $\tau_{n}$ for the Half-Plane Domain with Horizontal Reactions with $n=4$

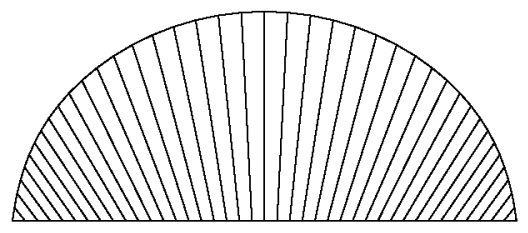

(a)

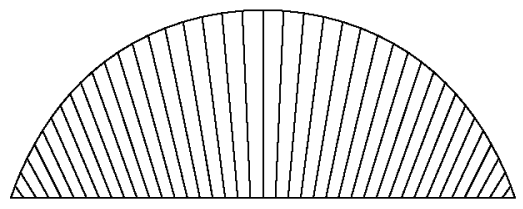

(b)

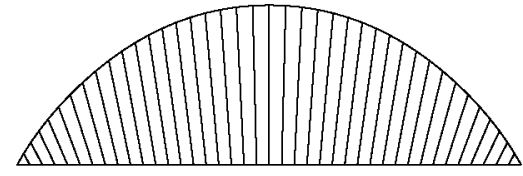

(c)

Fig. 5. AOH layouts for the half-plane domain with horizontal reactions: (a) $\phi=0.25$; (b) $\phi=0.50$; (c) $\phi=0.738$ 


$$
\begin{aligned}
\frac{d \mathcal{C}}{d P}= & 2 c_{e}+\frac{1}{2}\left[\left(k_{+}+k_{-}\right) \cdot \frac{d V_{\mathrm{opt}}^{\mathrm{AOH}, h}(\phi)}{d \phi}\right. \\
& \left.+\left(k_{+}-k_{-}\right) \cdot \frac{d \mathcal{M}(\phi)}{d \phi}\right] \frac{d \phi}{d P}=0
\end{aligned}
$$

subject to $P \geq P_{\min }$. From Eq. (23), $d \phi / d P=2 \mu / w L$. As $\mathcal{M}=-\phi w L^{2} / 2, d \mathcal{M} / d \phi=-w L^{2} / 2$. A simple but accurate approximation to Eq. (21) is

$$
\mathcal{V}_{\mathrm{opt}}^{\mathrm{AOH}, h}(\phi) \approx\left(0.9853-0.4653 \phi+0.2908 \phi^{2}\right) w L^{2}
$$

From Eqs. (25) and (26), the optimum $\phi$ for $\mathcal{C}$ can be obtained as

$$
\phi_{\mathrm{opt}}^{\mathcal{C}} \approx \frac{1.660 c_{+} \frac{L}{\mathcal{S}_{+}}-0.05966 c_{-} \frac{L}{\mathcal{S}_{-}}-3.439 \frac{c_{e}}{\mu}}{c_{+} \frac{L}{\mathcal{S}_{+}}+c_{-} \frac{L}{\mathcal{S}_{-}}}>0.738
$$

and from Eq. (23) the optimum $P$ is

$$
\begin{aligned}
P_{\mathrm{opt}}^{\mathcal{C}} & \approx \frac{1}{2} w L \frac{1}{\mu} \frac{(1.660-\mu) c_{+} \frac{L}{\mathcal{S}_{+}}-(0.05966+\mu) c_{-} \frac{L}{S_{-}}-3.439 c_{e}}{c_{+} \frac{L}{S_{+}}+c_{-} \frac{L}{S_{-}}} \\
& <P_{\min }
\end{aligned}
$$

This result is only useful to select appropriate layout and slenderness for a preliminary design in the case of structures with negligible self-weight; however, it depends on the relative sizes of the structure $\left(L / \mathcal{S}_{+}, L / \mathcal{S}_{-}\right)$. For large structures whose self-weight is of the order of the useful load, the self-weight has to be included in Eq. (23) and in the formulation of the problem Eq. (21). Hence, to get a true picture for real problems, the self-weight should be considered. Optimal geometry and horizontal reactions for other nonoptimal layout families can be estimated with this approach once the law for $\mathcal{V}(\phi)$ is established, as in Eq. (26).

\section{Conclusions}

The stress volume of the traditional parabolic arch with vertical hangers solution ( $\tau_{0}$ in Table 3 ) is approximately $17.3 \%$ greater than that of the solution with the layout proposed here, and the latter is approximately $1 \%$ greater that the best solution known to date, the solution obtained with simulated annealing in Table 1. Accounting with normal values of friction coefficient, the Michell number of the proposed layout can be reduced up to 0.85 , approximately $86 \%$ of the amount of the nonfrictional case, and only approximately $12.6 \%$ greater than the absolute optimum estimate computed by McConnel for the full-plane case, which can be considered an absolute lower limit. The layouts proposed in this paper are simpler than the proposed Michell nets by McConnel or Hemp, or that of the better solutions obtained with simulated annealing. From a practical point of view, this difference on shape is more meaningful than the small stress volume difference. Therefore, it seems reasonable to speak of near-optimal layouts.

There are some real bridges whose layouts resemble the $\mathrm{AOH}$ ones, at least in the use of oblique hangers [e.g., the Apollo bridge in Bratislava (Gabler 2006)]. The named network arch layout has a close relationship with the layouts proposed here (Tveit 2007). From a theoretical point of view, Pichugin et al. (2012, Fig. 2) report oblique hangers in the best solutions they obtain for the fixed boundary version of the problem. The important point is that the oblique-hangers design agrees with a classical rule of thumb: "lead the load toward the supports as straight as you can do it."

Interesting forward steps would be to introduce self-weight and to explore other instances of the bridge problem.

\section{Acknowledgments}

We are indebted to José Ignacio Hernando (Madrid) for his linear programming solutions, Wilfrid Gangbo for his valuable answers to our questions about his own research, and anonymous reviewers for their valuable criticism. We also thank Blanca Estevan for her search of bridges resembling the $\mathrm{AOH}$ layout. Many of the research work for this paper was carried out during the one-year stay of M. Vázquez Espí at CIMNE (Universidad Politécnica de Cataluña, Barcelona), financed by the Universidad Politécnica de Madrid. Thanks are due to Professor Eugenio Oñate and the CIMNE staff for their hospitality.

\section{References}

Antuña, J., and Vázquez Espí, M. (2012). "Existen problemas estructurales irresolubles? Una cuestión abierta." Informes de la Construcción, 64(525), 103-109.

Baker, W. F., Beghini, L. L., Mazurek, A., Carrion, J., and Beghini, A. (2013). "Maxwell's reciprocal diagrams and discrete Michell frames." Struct. Multidisc. Optim., 48(2), 267-277.

Bouchitté, G., Gangbo, W., and Seppecher, P. (2008). "Michell trusses and lines of principal action." Math. Models Methods Appl. Sci., 18(9), $1571-1603$

Bow, R. H. (1873). Economics of construction in relation to framed structures, E. \& F. N. Spon, London.

Cervera Bravo, J. (1989). "Tres teoremas fundamentales de la teoría del diseño de estructuras." Informes de la Construcción, 40(399), 57-66.

Cervera Bravo, J., and Vázquez Espí, M. (2011). "Galileo, Maxwell, Michell, Aroca: Measuring the structural efficiency." Structural Milestone in Architecture and Engineering. Int. Conf. on Research in Construction, IETcc-UPM, Madrid, Instituto de Ciencias de la Construcción (CSIC), 〈http://oa.upm.es/9931/) (Oct. 14, 2013).

Clausius, R. (1885). Über die Energievorräte der Natur und ihre Verwertung zum Nutzen der Menschheit, Verlag von Max Cohen \& Sohn, Bonn, Germany.

Cox, H. L. (1965). The design of structures of least weight, Pergamon, Oxford, U.K

Cross, H. (1936). "The relation of analysis to structural design." Trans. Am. Soc. Civ. Eng., 101(1), 1363-1374.

Darwich, W., Gilbert, M., and Tyas, A. (2010). "Optimum structure to carry a uniform load between pinned supports." Struct. Multidiscip. Optim. 42(1), 33-42.

de Miguel Rodríguez, J. L., and Aroca, R. (1974). "Trabajo estructural: un nuevo escalar de las estructuras." Ph.D. dissertation, ETS de Arquitectura de la Universidad Politécnica de Madrid, Madrid.

French, M. J. (1999). "A second law for structures: An insightfull approach to the design of plane frames." Trans. ASME, 66(3), 738-741.

Gabler, G. (2006). "Apollo-Donaubrücke in Bratislava." Der Stahlbau, $75(2), 138-144$.

Galilei, G. (1638). Discorsi e Dimostrazioni Matematiche, Elsevierii, Leiden, Holland.

Hemp, W. S. (1958). "Theory of structural design." Rep. 214, North Atlantic Treaty Organization, Advisory Group for Aeronautical Research \& Development, Paris.

Hemp, W. S. (1973). Optimum structures, Clarendon, Oxford, U.K.

Hemp, W. S. (1974). "Michell framework for uniform load between fixed supports." Eng. Optim., 1(1), 61-69.

Maxwell, J. C. (1870). "On reciprocal figures, frames and diagrams of forces." Scientific Papers II, Cambridge University Press, Cambridge, U.K., 160-202.

McConnel, R. E. (1974). "Least-weight framework for loads across span." J. Eng. Mech. Div., 100(EM5), 885-901.

Michell, A. G. M. (1904). "The limits of economy of material in framestructures." Philosophical Magazine S.6, 8(47), 589-597, 〈http:// habitat.aq.upm.es/gi/mve/dt/) (Oct. 14, 2013).

Owen, J. B. B. (1965). The analysis and design of light structures, Edward Arnold, London. 
Parkes, E. W. (1965). Braced frameworks: An introduction to the theory of structures, Pergamon Press, Oxford, U.K.

Pichugin, A. V., Tyas, A., and Gilbert, M. (2012). "On the optimality of Hemp's arch with vertical hangers." Struct. Multidisc. Optim. 46(1), 17-25.

Rozvany, G. I. N. (2011). "Comments on the authors' reply by T. Sokó and T. Lewiñski (2011) to a discussion by Vazquez and Cervera (2011) on their research paper (Sokó and Lewiñski 2010)." Struct. Multidisc. Optim., 44(5), 735-737.

Rozvany, G. I. N., and Sokó, T. (2012). "Exact truss topology optimization: Allowance for support costs and different permissible stresses in tension and compression-Extensions of a classical solution by Michell." Struct. Multidisc. Optim., 45(3), 367-376.
Srithongchai, S., and Dewhurst, P. (2003). "Comparisons of optimality criteria for minimum-weight dual material structures." Int. J. Mech. Sci., 45(11), 1781-1797.

Tveit, P. (2007). "An introduction to the optimal network arch." Struct. Eng. Int., 17(2), 184-187.

Vázquez Espí, C., and Vázquez Espí, M. (1997). "Discussion: Sizing, shape, and topology design optimization of trusses using genetic algorithm." J. Struct. Eng., 10.1061/(ASCE)0733-9445(1997)123:3(375), $375-376$.

Vázquez Espí, M. (2012). "On the allowance for support costs in PragerRozvany' optimal layout theory." Struct. Multidisc. Optim., 48(4), 847-852. 